\title{
X-ray-ultraviolet beam splitters for the Michelson interferometer
}

\author{
Franck Delmotte, Marie-Françoise Ravet, Françoise Bridou, Françoise Varnière, \\ Philippe Zeitoun, Sébastien Hubert, Laurent Vanbostal, and Gérard Soullie
}

\begin{abstract}
With the aim of realizing a Michelson interferometer working at $13.9 \mathrm{~nm}$, we have developed a symmetrical beam splitter with multilayers deposited on the front and back sides of a silicon nitride membrane. On the basis of the experimental optical properties of the membrane, simulations have been performed to define the multilayer structure that provides the highest reflectivity-transmission product. Optimized Mo-Si multilayers have been successfully deposited on both sides of the membrane by use of the ion-beam sputtering technique, with a thickness-period reproducibility of $0.1 \mathrm{~nm}$. Measurements by means of synchrotron radiation at $13.9 \mathrm{~nm}$ and at an angle of $45^{\circ}$ provide a reflectivity of $14.2 \%$ and a transmission of $15.2 \%$ for a $60 \% \mathrm{~s}$-polarized light, close to the simulated values. Such a beam splitter has been used for x-ray laser Michelson interferometry at $13.9 \mathrm{~nm}$. The first interferogram is discussed. (C) 2002 Optical Society of America
\end{abstract}

OCIS codes: $\quad 230.1360,230.4170,310.1860,310.6860,340.0340,340.7450$.

\section{Introduction}

Since their first realization around 15 years ago, $\mathrm{x}$-ray transmissive optics permit new investigations in x-ray optics applications. Now x-ray optics technology has evolved to the point at which it is possible to extend the applications field to x-ray-ultraviolet (X-UV) interferometry with the objective to characterize optics or dense plasmas. Indeed, in the study of laser-produced plasmas, optical interferometry has played a key role in absolute measurements of electron densities in a wide range of plasmas. ${ }^{1,2}$ But the maximum electron density accessible and the size of the probed plasmas are severely restricted by absorption and refraction of the probe beam. For these

F. Delmotte (franck.delmotte@iota.u-psud.fr), M.-F. Ravet, F. Bridou, and F. Varniere are with Groupe de Physique des Films Minces, Laboratoire Charles Fabry de l'Institut d'Optique, Centre National de la Recherche Scientifique, Unité Mixte de Recherche 8501, Centre Scientifique Bâtiment 503, 91403 Orsay CEDEX, France. P. Zeitoun, S. Hubert, and L. Vanbostal are with Laboratoire de Spectroscopie Atomique et Ionique, Centre National de la Recherche Scientifique, Unité Mixte de Recherche 8624, Université Paris-Sud, 91405 Orsay, France. G. Soullie is with Commissariat a l'Energie Atomique, BP 12, 91680, Bruyères-le-Châtel, France.

Received 2 January 2002; revised manuscript received 25 June 2002.

0003-6935/02/285905-08\$15.00/0

(C) 2002 Optical Society of America reasons there has existed a need to develop interferometers in the soft-x-ray range in which these effects can be mitigated. A grating or a purely reflective x-ray interferometric system, such as the Fresnel bimirror setup, has been discussed in the literature and has been used successfully.3,4 However, either technique lacks some of the advantages of standard interferometer geometries. In $1995 \mathrm{Da}$ Silva and collaborators developed an amplitudedivision x-ray Mach-Zehnder, successfully used to probe a dense plasma at $15.5 \mathrm{~nm} .{ }^{5}$ This experiment revealed internal residual stresses in X-UV beam splitters, ${ }^{6}$ which induce fringe-pattern deformations that can hamper the interpretation of plasmas' interferograms. So to reduce the fringe-pattern deformation, we chose to develop an X-UV Michelson interferometer consisting of only one beam splitter with its own internal stress operating at a $45^{\circ}$ incidence angle at $13.9 \mathrm{~nm}$.

Silicon nitride membranes have been widely used as a semitransparent substrate for x-ray transmissive optics in the wavelength range of $13-16 \mathrm{~nm}$ because of their relatively high transmission. ${ }^{5,7,8}$ Indeed, to realize a beam splitter in the soft-x-ray range, one can either deposit a multilayer on a membrane or deposit a multilayer on a silicon nitride layer and then etch the substrate's back side. In either case, the beam splitter can provide a good reflectivity from the coated side and a good transmission. Unfortunately, the reflectivity from the uncoated side is 


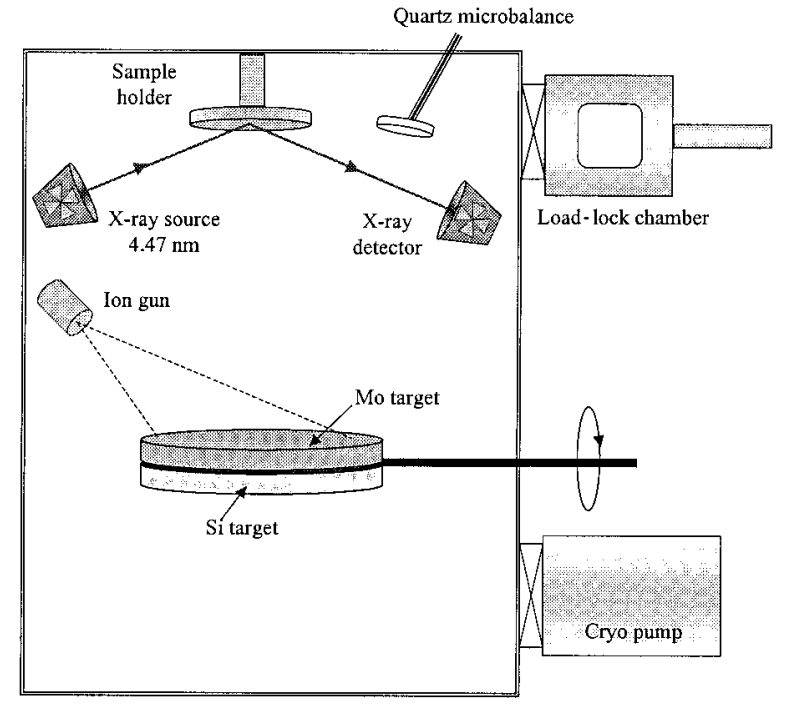

Fig. 1. Schematic diagram of the ultrahigh-vacuum deposition system.

low (typically less than 5\%) owing to the absorption in the silicon nitride membrane before and after the reflection on the multilayer coating. Consequently, this kind of beam splitter is not suited for the Michelson interferometer, in which good reflectivities from both sides of the beam splitter are required to prevent contrast losses between the two arms of the interferometer. One solution to this problem is to use free-standing multilayers as those described by Haga et al. ${ }^{9}$ However, the development of such a beam splitter is rather complex and involves many technological steps, leading to a high cost.

As an alternative, we propose a symmetrical beam splitter with multilayers deposited on the front and back sides of a silicon nitride membrane. In this paper we describe the simulation, development, and wavelength calibration of this new kind of beam splitter to be used in an X-UV Michelson interferometer.

\section{Experimental Technique}

Multilayers are deposited by an ion-beam sputtering technique, in a ultrahigh-vacuum chamber cryopumped to a base pressure of less than $10^{-6} \mathrm{~Pa}$ and equipped with a load-lock chamber (Fig. 1). The 5 -in.-diameter $(1 \mathrm{in} .=2.54 \mathrm{~cm})$ Mo and Si targets are successively ion beam sputtered by rotation of the target-holder system. We use a 3-cm Ion Tech ionbeam source with a mixture of $10 \% \mathrm{H}_{2}$ in Ar gas. Throughout the study, the ion-beam current and ion energy on the target are fixed at $30 \mathrm{~mA}$ and $650 \mathrm{eV}$, respectively. The samples are mounted on top of the chamber on a 2-in.-diameter sample holder. Deposited thicknesses are controlled by means of a quartz microbalance and an in situ x-ray reflectometer working at $4.47 \mathrm{~nm}(\mathrm{Cu} \mathrm{K} \alpha)$. The combination of these two techniques provides good accuracy on the period thickness of the multilayer.

The specular $\mathrm{x}$-ray reflectivity was measured $e x$ situ as a function of the incident angle with a reflec- tometer working with the $\mathrm{Cu} \mathrm{K} \alpha$ emission $(\lambda=$ $0.15405 \mathrm{~nm}$ ) delivered by a classical x-ray tube, equipped with a graphite rear monochromator and $40-\mu \mathrm{m}$ entrance and reception slits. The angular resolution of the reflectometer is better than 0.001 deg. The reflectivity curve is obtained by our varying the grazing angle while tracking the reflected beam. This kind of curve allows the determination of the parameters of the stack such as the complex indices, the thickness of each layer, and the interfacial roughnesses. After computation of theoretical reflectivities starting with given parameters, the experimental reflectivity curve can be fitted by a trialand-error method. ${ }^{10}$

An Akashi Topcon transmission electron microscope operated at $200 \mathrm{kV}$ with a $\mathrm{LaB}_{6}$ filament was used for transmission electron microscopy (TEM) studies. Cross-sectional specimens for TEM were mechanically polished to $80 \mu \mathrm{m}$ and then dimpled in the center to $20 \mu \mathrm{m}$. This was followed by further thinning to electron transparency by $\mathrm{Ar}^{+}$-ion milling at $6 \mathrm{kV}$ in a cooling stage.

Reflectivity and transmission measurements in the energy range of 65-150 eV have been performed at the Super ACO synchrotron facility (Orsay, France) on the SA23 beamline. The line is equipped with a 750-lines/mm toroidal grating monochromator and a soft-x-ray reflectometer with an International Radiation Detectors, Inc. AXUV100 silicon diode as detector. The monochromator resolution is approximately $0.7 \mathrm{eV}$ at $100 \mathrm{eV}$ and the degree of $s$ polarization is approximately $60 \%$.

\section{Results and Discussion}

\section{A. Preliminary Study: Membrane Charaterization}

In this study we use commercially available silicon nitride membranes provided by Silson Ltd. The silicon frame size is $10 \mathrm{~mm} \times 10 \mathrm{~mm}$, and the membrane size is $5 \mathrm{~mm} \times 5 \mathrm{~mm}$. The $\mathrm{Si}_{3} \mathrm{~N}_{4}$ thickness is in the range of $80-100 \mathrm{~nm}$.

Silicon nitride membranes have been first characterized by x-ray grazing-angle reflectometry to determine the thickness and the optical constant of $\mathrm{Si}_{3} \mathrm{~N}_{4}$. The experimental data and the fit of these data are shown on Fig. 2. The fit gives a thickness of $88.5 \mathrm{~nm}$ and an index $\delta$ of $9.6 \times 10^{-6}$, i.e., approprimately $85 \%$ of the tabulated value for bulk $\mathrm{Si}_{3} \mathrm{~N}_{4}$.

Figure 3 shows the transmission of the $\mathrm{Si}_{3} \mathrm{~N}_{4}$ membrane measured at $45^{\circ}$ in the $65-150-\mathrm{eV}$ energy range. The high transmission at $89.2 \mathrm{eV}$ (more than $35 \%$ ) confirms that these membranes can be used as support for beam splitters. We have also plotted on Fig. 3 the simulated transmission of a single film of $\mathrm{Si}_{3} \mathrm{~N}_{4}$, with a 88.5-nm thickness and a density of 2.92 $\mathrm{g} / \mathrm{cm}^{3}$ (85\% of the bulk $\mathrm{Si}_{3} \mathrm{~N}_{4}$ density). We note an energy shift in the Si absorption edge between simulation and experimental data. This shift is approximately $3.4 \mathrm{eV}$ and is interpreted as a consequence of the nature of chemical bonding in $\mathrm{Si}_{3} \mathrm{~N}_{4}$, which is not taken into account in the simulation. Apart from this shift, the simulated curve is in good agreement 


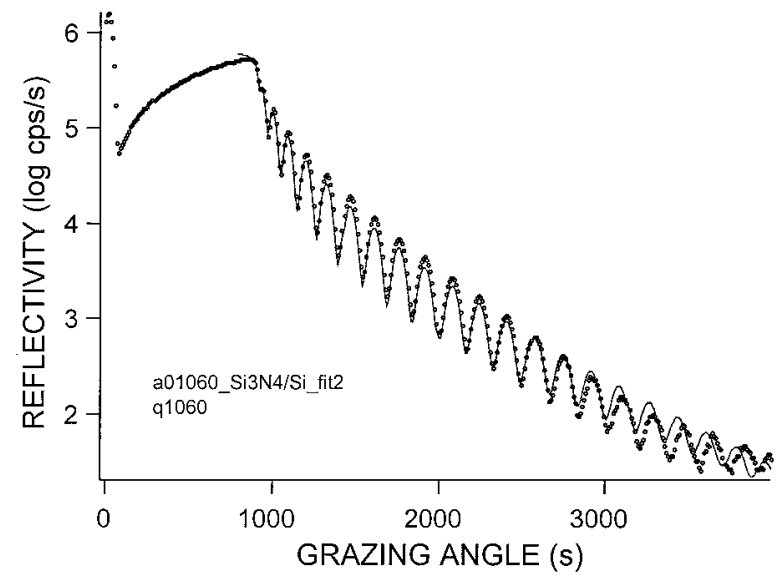

Fig. 2. X-ray grazing-angle reflectometry curve measured at $0.154 \mathrm{~nm}$ on a silicon nitride membrane (circles) and fitted curve (solid curve) obtained with a thickness of $88.5 \mathrm{~nm}$ and an index $\delta$ of $9.6 \times 10^{-6}$ for the silicon nitride. cps, counts per second.

with the experimental data, indicating that the parameters chosen for the simulation are relevant. So we have decided to use this membrane model for the beam splitter simulations.

\section{B. Optimization of the Beam Splitter Structure by Way of Simulation}

In the wavelength range of interest for the x-ray laser interferometry applications (around $13.9 \mathrm{~nm}$ ), Mo and $\mathrm{Si}$ are common materials that present a good optical contrast, so we choose these materials to perform the multilayer coating.

As explained in the introduction of this paper, we have developed a new kind of beam-splitter structure with multilayers deposited on the front and back sides of the silicon nitride membrane. The beamsplitter structure is shown in Fig. 4; we call it a "both-sides-coated $\mathrm{Si}_{3} \mathrm{~N}_{4}$ membrane." Owing to the symmetry of this beam-splitter structure, the reflectivity from the back side should be the same as the

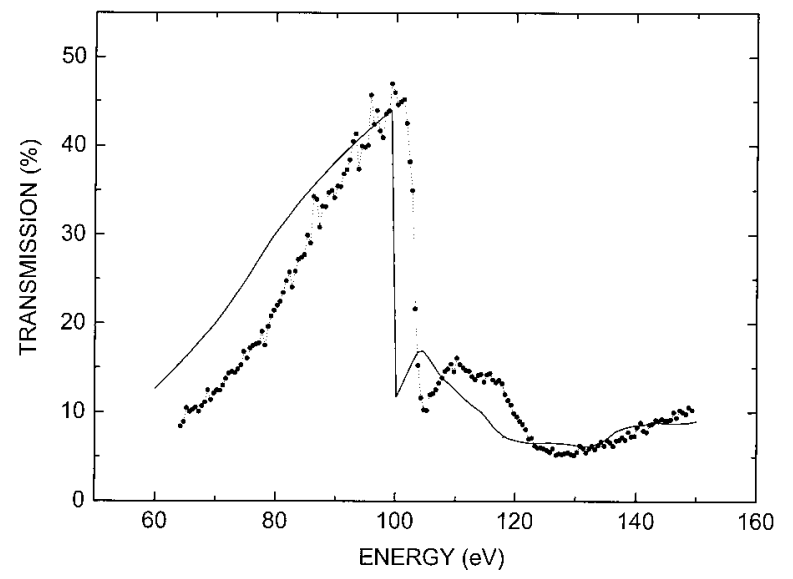

Fig. 3. Transmission of the $\mathrm{Si}_{3} \mathrm{~N}_{4}$ membrane at $45^{\circ}$ in the $65-$ 150-eV energy range: Circles represent the experimental values, and the solid curve is the simulated curve for a silicon nitride film with a 88.5-nm thickness and a density of $2.92 \mathrm{~g} / \mathrm{cm}^{3}$.

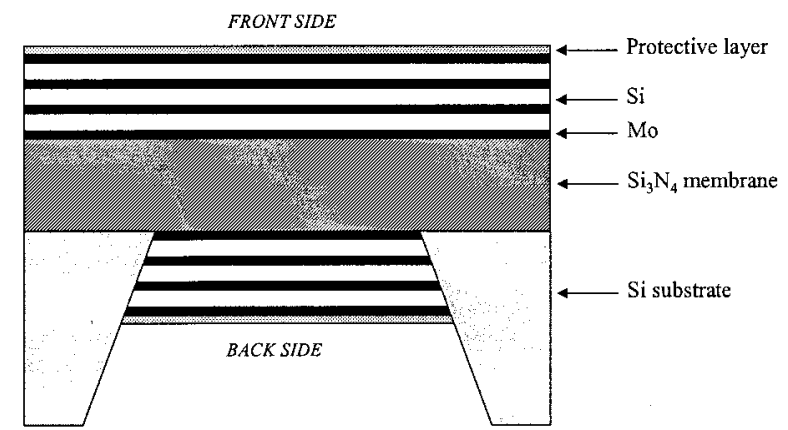

Fig. 4. Schematic diagram of a both-sides-coated silicon nitride membrane.

reflectivity from the front side. We have simulated this kind of beam splitter to optimize the relative thicknesses of Mo in the period $\left(\gamma=\mathrm{th}_{\mathrm{Mo}} / \mathrm{th}_{\mathrm{Si}}+\mathrm{th}_{\mathrm{Mo}}\right)$ and the number of periods on each side $\left(N_{P}\right)$. All simulations were made in the case of $s$-polarized light under an incidence angle of $45^{\circ}$ fixed by the interferometer design. In fact, as the angle of incidence is the Brewster angle, the reflectivity for $p$ polarization is very low (typically, 100 times lower than for $s$ polarization). So, when mounted in a Michelson interferometer, the beam splitter will be effective only for the $s$ component of the x-ray source.

We have calculated the variation of the reflectivity-transmission product $(R \times T$ product) as a function of the relative thickness of Mo in the period with optimized $N_{P}$ for each thickness and with a period thickness of $10.2 \mathrm{~nm}$. Results are optimized with $\gamma$ values in the range of 0.2-0.3. Figure 5 shows the variation of the $R \times T$ product as a function of the number of Mo-Si periods on each side with $\gamma$ fixed at 0.25: Approximately $3 \%$ of the $R \times T$ product can be obtained with $N_{P}$ equal to 4 or 5 .

On Fig. 6 we compare the reflectivities of a bothsides-coated (curve a) and a one-side-coated (curves b and c) $\mathrm{Si}_{3} \mathrm{~N}_{4}$ membrane. In this latter case, we have calculated the reflectivity from the coated side (curve b) and the reflectivity from the uncoated side (curve c). For a both-sides-coated $\mathrm{Si}_{3} \mathrm{~N}_{4}$ membrane, the re-

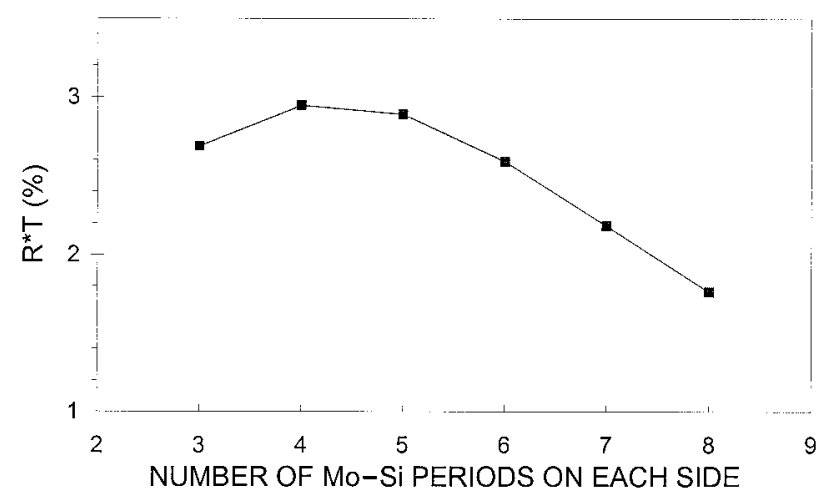

Fig. 5. Variation of the reflectivity-transmission product as a function of the number of Mo-Si periods on each side of the membrane. The relative thickness of Mo in the period $(\gamma)$ was fixed at 0.25 . 


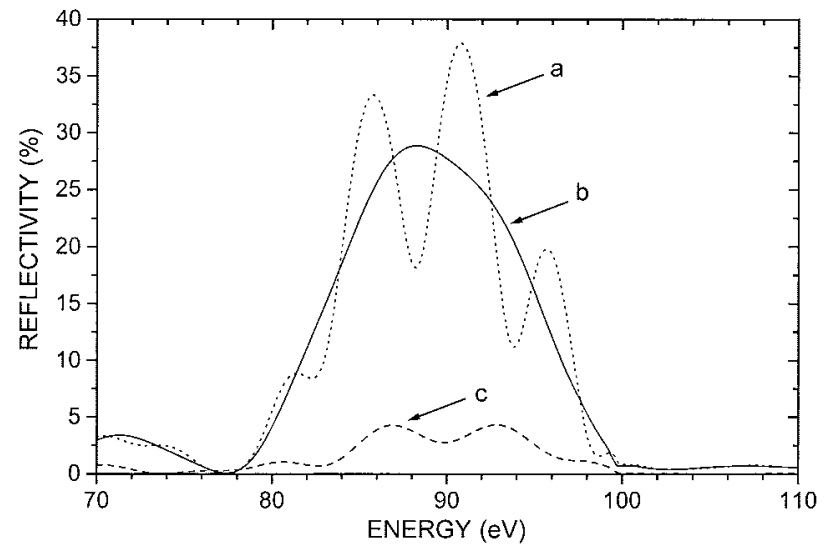

Fig. 6. Simulated reflectivities for a both-sides-coated $\mathrm{Si}_{3} \mathrm{~N}_{4}$ membrane (curve a) and for a one-side-coated $\mathrm{Si}_{3} \mathrm{~N}_{4}$ membrane with light coming on the coated side (curve b) or on the uncoated side (curve c).

flectivity from the front and back sides would be the same owing to the symmetry of the beam-splitter structure. The oscillations that appear on the reflectivity spectra a and c are due to the Fabry-Perot effect in the stack (the $\mathrm{Si}_{3} \mathrm{~N}_{4}$ membrane acts as a cavity). We also note that at the energy of interest $(89.2 \mathrm{eV}$ for a wavelength of $13.9 \mathrm{~nm})$, the both-sidescoated membrane presents a reflectivity of $25.8 \%$, which is close to the reflectivity from the front side of the one-side-coated membrane $(28.4 \%)$ without the inconvenience of a very low reflectivity $(2.9 \%)$ from the back side. Calculations of the $R \times T$ product indicate an enhancement by a factor of 5 on the throughput by use of the both-sides-coated membrane. These results clearly show that the bothsides-coated $\mathrm{Si}_{3} \mathrm{~N}_{4}$ membrane is well suited for Michelson interferometry applications.

\section{Fabrication of the Beam Splitter: Multilayer Deposition and Control}

Before coating the $\mathrm{Si}_{3} \mathrm{~N}_{4}$ membrane, we have to be sure of the quality and thicknesses of the deposited layers. For this study, we have deposited Mo-Si multilayers with Mo and Si thicknesses deduced from simulations on test samples (silicon or float glass substrates) with $N_{P}$ equal to 5 . These samples were first analyzed by grazing x-ray reflectometry at 0.154 $\mathrm{nm}$.

Figures 7(a) and 7(b) show the experimental curves and the fitted curves for two float glass substrates coated in the same conditions but not at the same time. The parameters determined by these fits are given in Tables 1 and 2 , respectively. We note that the complex indices of Mo and Si layers at $0.154 \mathrm{~nm}$ are close to the tabulated ones ${ }^{11}$ :

$$
\begin{array}{ll}
\delta_{\mathrm{Si}}=7.6 \times 10^{-6}, & \delta_{\mathrm{Mo}}=28.6 \times 10^{-6}, \\
\beta_{\mathrm{Si}}=0.17 \times 10^{-6}, & \beta_{\mathrm{Mo}}=1.87 \times 10^{-6} .
\end{array}
$$

These results suggest that deposited Mo and Si materials are pure and dense. These fits also indicate
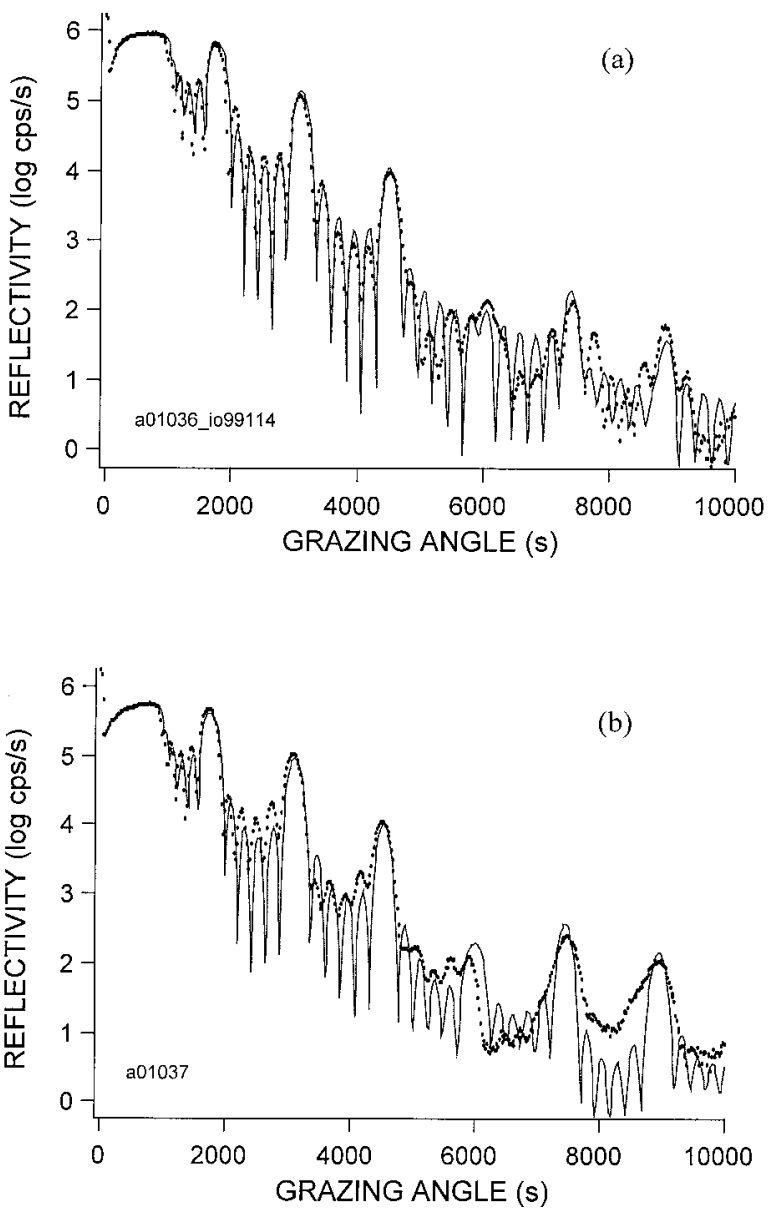

Fig. 7. X-ray grazing-angle reflectometry curves: Experimental data measured at $0.154 \mathrm{~nm}$ on multilayer-coated float glass sample (circles) and fitted curve (solid curve). (a) and (b) correspond to two different samples deposited in the same condition but not at the same time.

that the interfacial roughness estimated by x-ray reflectometry is approximately $0.7 \mathrm{~nm}$.

TEM and high-resolution TEM (HRTEM) measurements are in good agreement with these results. The photographs shown in Figs. 8(a) and 8(b) reveal dense and continuous Mo and Si layers with welldefined interfacial regions. The interface between the first Mo layer and the Si substrate is rather rough probably owing to the oxide layer of the silicon substrate that had not been removed before deposition. The interfaces of the additional Mo layers are sharper and clearly show the effect of smoothening of the interfaces after only one period deposited. Moreover, a high-resolution TEM analysis indicates that the Si and Mo layers are amorphous [Fig. 8(b)]. A polycrystalline structure of the Mo layers has often been reported in the literature in the case of magnetron-sputtering deposition. ${ }^{7,12-15}$ However, the structure of Mo layers has been found to depend on the deposition method used, and amorphous Mo layers have been deposited by e-beam evaporation and ion-beam sputtering. ${ }^{12}$

One key for the success of the beam splitter is the 
Table 1. Results of the Fit of an X-Ray Grazing-Angle Reflectometry Curve Measured on a Multilayer-Coated Float Glass Sample ${ }^{a}$

\begin{tabular}{lcccc}
\hline \multicolumn{1}{c}{ Material } & Thickness $(\mathrm{nm})$ & Roughness $(\mathrm{nm})$ & Index $\delta$ & Absorption $\beta$ \\
\hline Substrate, float glass & $\infty$ & 0.4 & $7.7 \times 10^{-6}$ & $0.13 \times 10^{-6}$ \\
Layer 1, Mo & 2.73 & 0.7 & $26 \times 10^{-6}$ & $1.9 \times 10^{-6}$ \\
Layer 2, Si & 8.1 & 0.6 & $7.5 \times 10^{-6}$ & $0.13 \times 10^{-6}$ \\
Layer 3, Mo & 2.73 & 0.7 & $26 \times 10^{-6}$ & $1.9 \times 10^{-6}$ \\
$\vdots$ & 3 & 0.3 & $7.5 \times 10^{-6}$ & $0.13 \times 10^{-6}$ \\
Layer 12, Si & 3 & & & \\
\hline
\end{tabular}

${ }^{a}$ See Fig. 7(a).

reproductibility of the multilayer deposition on both sides of the membrane. The association of in situ reflectometry and in situ quartz microbalance allows us to control the thickness of the period within $0.1 \mathrm{~nm}$ between two depositions as illustrated in Figs. 7(a) and 7(b) and in Tables 1 and 2.

It is also essential to minimize the tensile stress in the beam splitter after the multilayer deposition (approximately 40 to $50 \mathrm{MPa}$ ). ${ }^{9,16}$ Ion-beam-deposited multilayers are known to be highly stressed in a compressive mode: The stress of a Mo-Si multilayer similar to those used in the beam splitter (with $N_{P}$ equal to 5) has been calculated from the measurements of a Si substrate curvature before and after multilayer deposition by use of Stoney's formula and is approximately $-810 \mathrm{MPa}$. So we choose a highly tensile $\mathrm{Si}_{3} \mathrm{~N}_{4}$ membrane: By varying the period thickness and number of period $N_{P}$, we can adjust the total stress value so that it remains in tensile mode. In the case of $N_{P}$ equal to 5 , we found that beam splitters wrinkle when the multilayer coating has a period thickness greater than $10.7 \mathrm{~nm}$, whereas they remain flat when the period thickness is approximately $10 \mathrm{~nm}$. In the case of $N_{P}$ equal to 4 , the beam splitters remain flat whatever the period thickness is, in the range of 10-10.7 $\mathrm{nm}$.

\section{Test and Calibration of the Beam Splitter}

We have first measured grazing x-ray reflectivity of a both-sides-coated membrane at $0.154 \mathrm{~nm}$ to verify that the substrate nature (i.e., the silicon nitride membrane) does not significantly modify the multilayer quality. The measured data are shown on Fig. 9. The fit of these data is rather complex, so it has been made in two steps. First, we have determined the complex indices of materials by fitting the data coming from measurements on float glass witness samples that were coated at the same time as the beam splitter (one for each side). Then we have fixed the value of the complex indices, and we have fitted the data measured on the beam splitter by varying thicknesses and interfacial roughnesses. The fitted curve is shown on Fig. 9, and the results of this fit are given in Table 3. This result confirms that we have deposited very similar multilayers on both sides (period thickness of 9.9 and $10 \mathrm{~nm}$ ) and indicates that the interfacial roughness in the multilayers deposited on the membrane is not higher than those measured on float glass samples (Tables 1 and 2).

Beam splitters with $N_{P}$ equal to 4 and 5 have also been tested on a synchrotron radiation source around the wavelength $13.9 \mathrm{~nm}$ (89.2 eV in energy). All transmission and reflectivity measurements were made with an incidence angle of $45^{\circ}$. On Fig. 10(a) the transmission of a beam splitter with a $N_{P}$ of 4 and a period thickness of $10.7 \mathrm{~nm}$ is plotted as a function of x-ray energy. We note that two absorption edges appear on this curve: The first at approximately 100 $\mathrm{eV}$ is related to the absorption edge of silicon in the deposited layers, and the second at approximately $104 \mathrm{eV}$ is related to the absorption edge of silicon in the silicon nitride layer, as reported on Fig. 3. The transmission value measured at $89 \mathrm{eV}$ is $16.8 \%$ in this case. The reflectivity curve measured on the same beam splitter is plotted on Fig. 10(b). As predicted by simulations, oscillations appear because of the Fabry-Perot effect in the $\mathrm{Si}_{3} \mathrm{~N}_{4}$ membrane. At $89 \mathrm{eV}$ the measured reflectivity is $10.5 \%$. These results show that, even with an unoptimized period thickness, this kind of beam splitter provides satisfying transmission and reflectivity.

As expected, the reflectivity of a beam splitter with a $N_{P}$ of 5 and a period thickness of $10 \mathrm{~nm}$ is higher and the transmission is somewhat lower: Measurements at $89 \mathrm{eV}$ give $14.2 \%$ for reflectivity and $15.2 \%$

Table 2. Results of the Fit of an X-Ray Grazing-Angle Reflectometry Curve Measured on a Multilayer-Coated Float Glass Sample ${ }^{b}$

\begin{tabular}{lcccc}
\hline \multicolumn{1}{c}{ Material } & Thickness $(\mathrm{nm})$ & Roughness $(\mathrm{nm})$ & Index $\delta$ & Absorption $\beta$ \\
\hline Substrate, float glass & $\infty$ & 0.4 & $7.7 \times 10^{-6}$ & $0.13 \times 10^{-6}$ \\
Layer 1, Mo & 2.65 & 0.6 & $25 \times 10^{-6}$ & $1.9 \times 10^{-6}$ \\
Layer 2, Si & 8.1 & 0.35 & $7.4 \times 10^{-6}$ & $0.13 \times 10^{-6}$ \\
Layer 3, Mo & 2.65 & 0.6 & $25 \times 10^{-6}$ & $1.9 \times 10^{-6}$ \\
$\vdots$ & 3 & 0.3 & $7.4 \times 10^{-6}$ & $0.13 \times 10^{-6}$ \\
Layer 12, Si & 3 & & & \\
\hline
\end{tabular}

${ }^{b}$ See Fig. 7(b). 

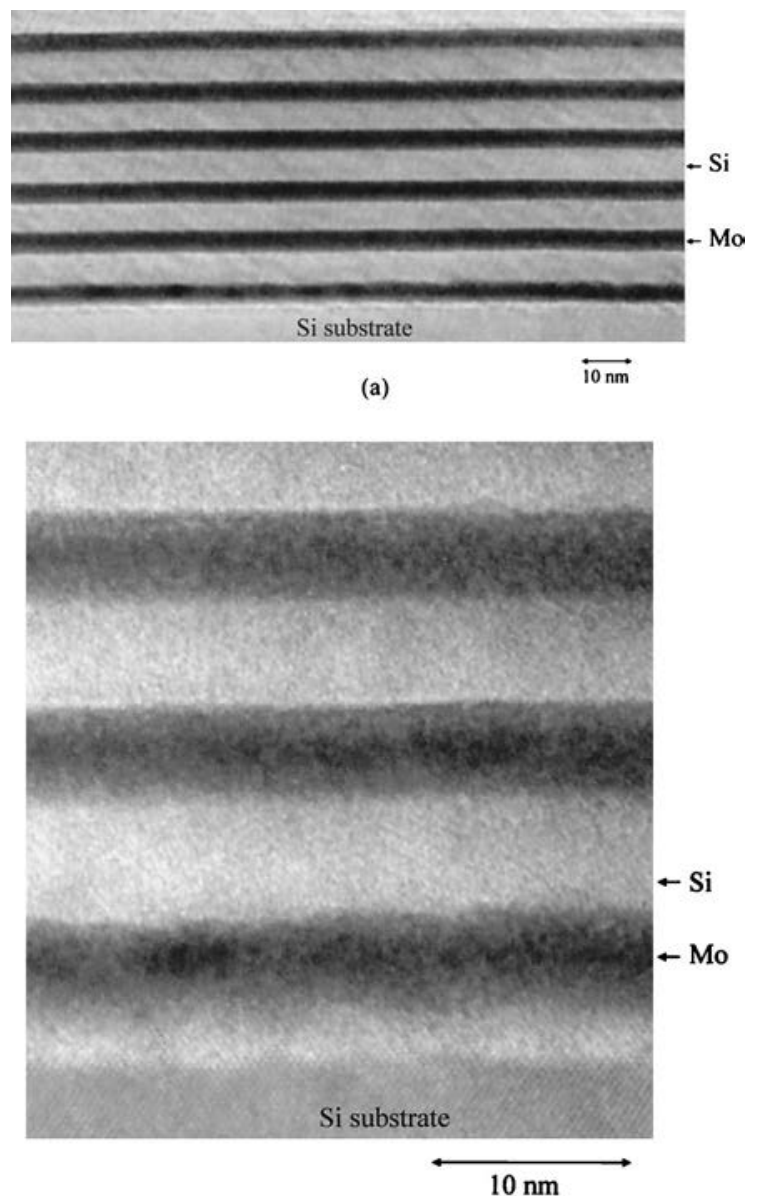

(b)

Fig. 8. TEM and high-resolution TEM pictures of a Mo-Si multilayer.

for transmission. From these values, and assuming a degree of $s$ polarization of $60 \%$ on the synchrotron beamline that we have used, we can estimate that reflectivity and transmission for $s$-polarized $\mathrm{x}$ rays are, respectively, approximately $23 \%$ and $13 \%$, which

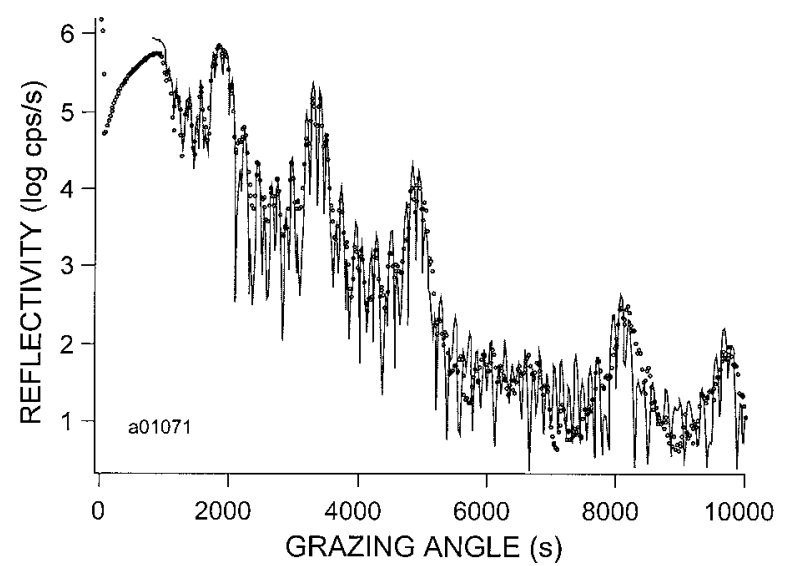

Fig. 9. X-ray grazing-angle reflectivity curves: experimental data measured on a both-sides-coated membrane at $0.154 \mathrm{~nm}$ (circles) and fitted curve (solid curve). means that the $R \times T$ product is approximately $3 \%$ for this beam splitter. These values are very close to the predicted ones (see Figs. 5 and 6) and show that the deposition of high-quality multilayers on silicon nitride membrane has been achieved.

Finally, we have also measured the reflectivity of the back side of the beam splitter. On Fig. 11 we compare the reflectivity of the front side of a beam splitter to the reflectivity of the back side of another beam splitter deposited in the same conditions $\left(N_{P}\right.$ of 5 and period thickness of $10 \mathrm{~nm}$ ). Although the period thicknesses are quite the same, a shift appears in the Fabry-Perot oscillations. This shift could be interpreted either as a variation of the silicon nitride membrane thickness between the two beam splitters or as a variation of the relative thickness of Mo $(\gamma)$ between the two multilayers.

Figure 11 shows that the reflectivity of the back side is higher than the reflectivity of the front side (26\% against $20 \%$ if we compare the peak value). These features have already been reported by several authors ${ }^{8,9}$ and have been related to the difference of roughness of the silicon nitride membrane's front side and back side (the back side's roughness is lower). Preliminary atomic force microscopy analysis have been done on both sides of our beam splitter and clearly show that the front side is much rougher than the back side. Further studies are in progress in order to know the real effect of this roughness on the beam-splitter performances and light polarization.

\section{Conclusion}

We have calculated and developed a new kind of $\mathrm{X}-\mathrm{UV}$ beam splitter with symmetrical properties as required for an $\mathrm{X}-\mathrm{UV}$ Michelson interferometer. Optimized Mo-Si multilayers have been successfully deposited on both sides of a commercially available silicon nitride membrane by use of the ion-beam sputtering technique. The thickness-period reproductibility is $0.1 \mathrm{~nm}$ and allows us to deposit similar multilayers on both sides of the membrane. Grazing $\mathrm{x}$-rays reflectometry at $0.154 \mathrm{~nm}$ and TEM analysis show that the multilayers are made of dense, continuous, and amorphous Mo and Si layers. The interfacial roughness estimated by $x$-ray reflectometry is approximately $0.7 \mathrm{~nm}$ and seems to be independent of the substrate type (float glass samples or silicon nitride membranes).

Synchrotron radiation measurements at $13.9 \mathrm{~nm}$ provide a reflectivity of $14.2 \%$ and a transmission of $15.2 \%$ for a $60 \% s$-polarized light. From these measurements we can deduce that the $R \times T$ product of the beam splitter is close to the simulated value of $3 \%$. The reflectivity of the back side has been measured to be somewhat higher than that of the front side. This phenomenon seems to be related to the roughness of the beamsplitter's front side, which has been found to be higher than the roughness of the back side, as measured by atomic force microscopy.

Finally, these beam splitters have been used in a Michelson interferometer for an experiment devoted for the first time, to our knowledge, to Fourier spec- 
Table 3. Results of the Fit of an X-Ray Grazing-Angle Reflectometry Curve Measured on a Both-Side-Coated Silicon Nitride Membrane ${ }^{a, b}$

\begin{tabular}{|c|c|c|c|c|}
\hline Material & Thickness (nm) & Roughness (nm) & Index $\delta$ & Absorption $\beta$ \\
\hline $\begin{array}{l}\text { Layer } 24, \mathrm{Si} \\
\vdots\end{array}$ & 3 & 0.48 & $7.4 \times 10^{-6}$ & $0.13 \times 10^{-6}$ \\
\hline Layer 15, Mo & 2.5 & 0.45 & $25 \times 10^{-6}$ & $1.8 \times 10^{-6}$ \\
\hline Layer 14, Si & 7.4 & 0.48 & $7.4 \times 10^{-6}$ & $0.13 \times 10^{-6}$ \\
\hline Layer 13, Mo & 2.5 & 0.45 & $25 \times 10^{-6}$ & $1.8 \times 10^{-6}$ \\
\hline $\mathrm{Si}_{3} \mathrm{~N}_{4}$ membrane & 88.5 & 0.18 & $9.5 \times 10^{-6}$ & $0.25 \times 10^{-6}$ \\
\hline Layer 1, Mo & 2.5 & 0.71 & $26 \times 10^{-6}$ & $1.9 \times 10^{-6}$ \\
\hline Layer $2, \mathrm{Si}$ & 7.5 & 0.7 & $7.5 \times 10^{-6}$ & $0.13 \times 10^{-6}$ \\
\hline $\begin{array}{l}\text { Layer } 3, \text { Mo } \\
:\end{array}$ & 2.5 & 0.71 & $26 \times 10^{-6}$ & $1.9 \times 10^{-6}$ \\
\hline Layer $12, \mathrm{Si}$ & 3 & 0.71 & $7.5 \times 10^{-6}$ & $0.13 \times 10^{-6}$ \\
\hline
\end{tabular}

${ }^{a}$ See Fig. 9.

${ }^{b}$ Layers 1 to 12 are on the front side, and layers 13 to 24 are on the back side of the silicon nitride membrane.

troscopy linewidth measurement of a Ni-like silver soft-x-ray laser source operating at $13.9 \mathrm{~nm}$ by Fourier spectroscopy. ${ }^{17}$ As shown on Fig. 12, the interferogram obtained with a no-difference path between the two arms presents a contrast ranging from $76 \%$ to $92 \%$, over the whole aperture of the beam splitter (5 $\mathrm{mm} \times 5 \mathrm{~mm}$ ). However, we note that large deformations of fringes arise from residual stresses both in the $\mathrm{Si}_{3} \mathrm{~N}_{4}$ membrane and in the Mo-Si multilayer
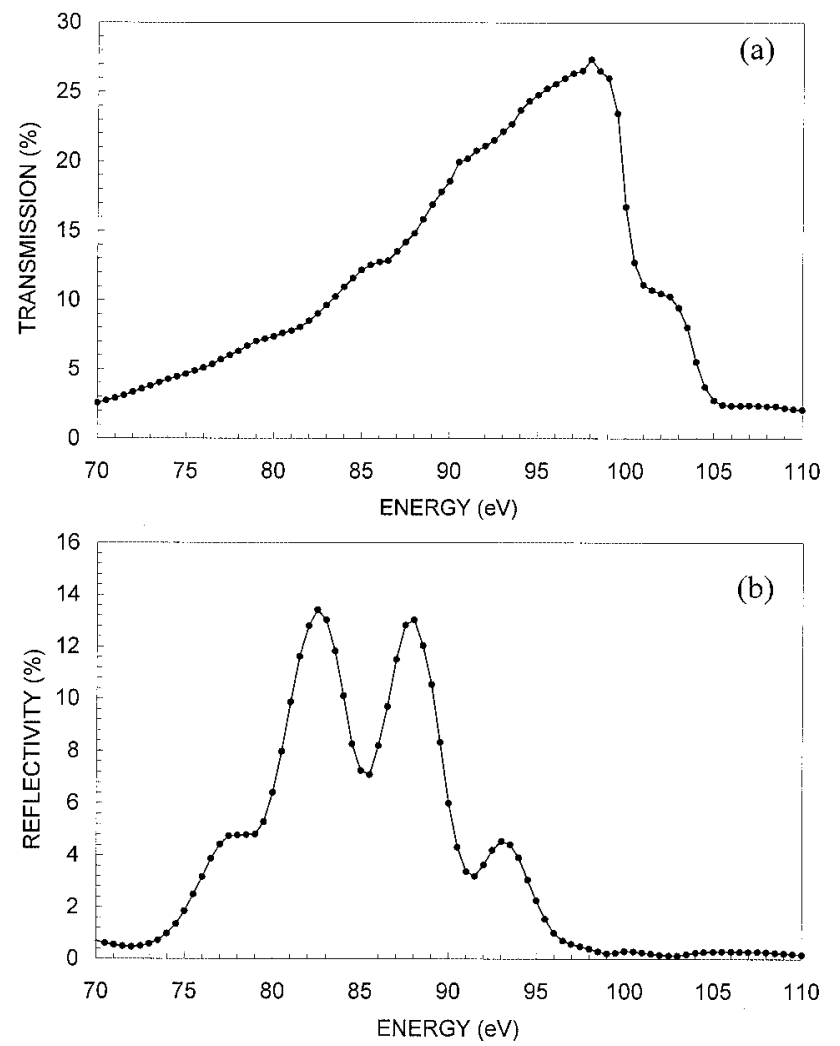

Fig. 10. Experimental transmission (a) and reflectivity (b) measured on a both-sides-coated membrane versus x-ray energy. $\mathrm{X}$ rays were coming on the front side. The angle of incidence was $45^{\circ}$ and the degree of $s$ polarization was approximately $60 \%$. The multilayers have four periods and a period thickness of $10.7 \mathrm{~nm}$.

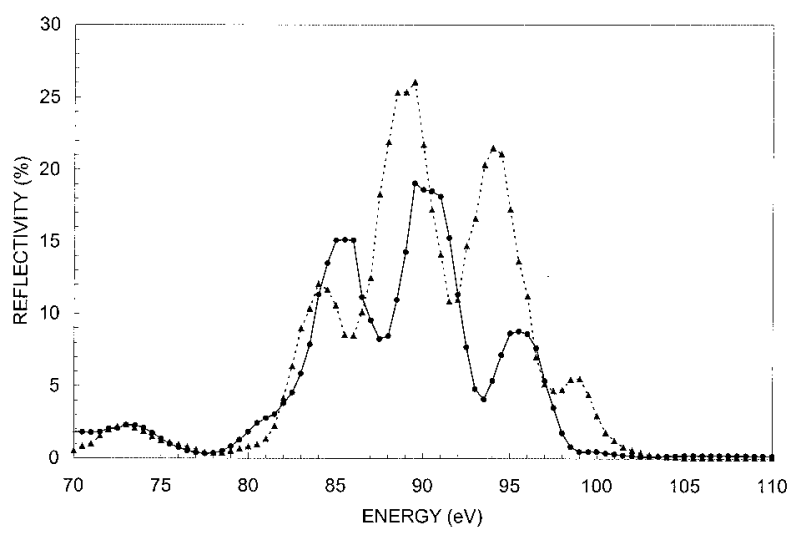

Fig. 11. Experimental reflectivities measured on two both-sidescoated membranes versus $\mathrm{x}$-ray energy, with $\mathrm{x}$ rays coming on the front side (circles) and on the back side (triangles). The angle of incidence was $45^{\circ}$, and the degree of $s$ polarization was approximately $60 \%$. The multilayers have five periods and a period thickness of $10 \mathrm{~nm}$.

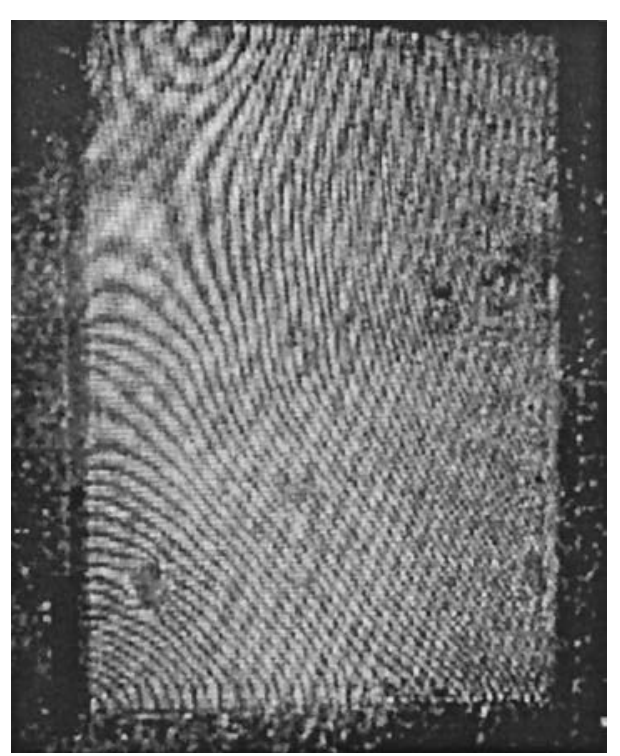

Fig. 12. Interferogram (screen shot) recorded at $13.9 \mathrm{~nm}$ with the Michelson interferometer with a zero-difference path difference. 
coating. To prevent this kind of fringe-pattern deformation, we are performing further studies to improve the x-ray beam splitter's flatness.

The authors thank Philippe Troussel from Commissariat a l'Energie Atomique, Bruyères-le-Châtel for the access to the reflectometer of the SA23 beamline at the Laboratoire pour l'Utilisation du Rayonnement Electromagnétique, Orsay. Financial support for this work was partially provided by the Centre National de la Recherche Scientifique program Traitement de surfaces et dépôt de couches minces.

\section{References}

1. G. Charatis, G. E. Busch, C. L. Shepard, P. M. Campbell, and M. D. Rosen, "Hydrodynamic aspects of selenium x-ray laser targets," J. Phys. C 6, 89-98 (1986).

2. M. K. Prasad, K. G. Estabrook, J. A. Harte, R. S. Craxton, R. A. Bosch, G. E. Busch, and J. S. Kollin, "Holographic interferograms from laser fusion code simulations," Phys. Fluids B 4, 1569-1575 (1992).

3. S. Chakrabarti, D. M. Cotton, J. S. Vickers, and B. C. Bush, "Self-compensating, all-reflection interferometer," Appl. Opt. 33, 2596-2602 (1994).

4. J. Svatos, D. Joyeux, D. Phalippou, and F. Polack, "Soft-x-ray interferometer for measuring the refractive index of materials," Opt. Lett. 18, 1367-1369 (1993).

5. L. B. Da Silva, T. W. Barbee, Jr., R. Cauble, P. Celliers, D. Ciarlo, S. Libby, R. A. London, D. Matthews, S. Mrowka, J. C. Moreno, D. Ress, J. E. Trebes, A. S. Wan, and F. Weber, "Electron density measurements of high density plasmas using soft X-ray laser interferometry,” Phys. Rev. Lett. 74, 3991-3994 (1995).

6. P. Celliers, F. Weber, L. B. Da Silva, T. W. Barbee, Jr., R. Cauble, A. S. Wan, and J. C. Moreno, "Fringe formation and coherence of a soft-x-ray laser beam illuminating a MachZehnder interferometer," Opt. Lett. 20, 1907-1909 (1995).

7. D. G. Stearns, N. M. Ceglio, A. M. Hawryluk, M. B. Stearns, A. K. Petford-Long, C. H. Chang, K. Danzmann, M. Kuhne, P. Muller, and B. Wende, "TEM and X-ray analysis of multilayer mirrors and beamsplitters," in Multilayer Structures and Lab- oratory X-Ray Laser Research, N. M. Ceglio and P. Dhez, eds., Proc. SPIE 688, 91-98 (1987).

8. N. M. Ceglio, "Revolution in X-ray optics," J. X-Ray Sci. Technol. 1, 7-78 (1989).

9. T. Haga, M. C. K. Tinene, A. Ozawa, Y. Utsumi, S. Itabashi, T. Ohkubo, and M. Shimada, "Fabrication of semitransparent multilayer polarizer and its application to soft x-ray ellipsometer," in Ultraviolet and X-Ray Detection, Spectroscopy, and Polarimetry III, S. Fineschi, B. E. Woodgate, and R. A. Kimble, eds., Proc. SPIE 3764, 13-27 (1999).

10. F. Bridou and B. Pardo, "Automatic characterization of layers stacks from reflectivity measurements. Application to the study of the validity conditions of the grazing X-rays reflectometry," J. Opt. (Paris) 21, 183-191 (1990).

11. B. L. Henke, E. M. Gullikson, and J. C. Davis, "X-ray interactions: photoabsorption, scattering, transmission, and reflection at $\mathrm{E}=50-30000 \mathrm{eV}, \mathrm{Z}=1-92$," At. Data Nucl. Data Tables 54, 181-342 (1993) or http://www-cxro.lbl.gov.

12. S. Ogura, M. Niibe, Y. Watanabe, M. Hayashida, and T. Iizuka, "Comparison among multilayer soft X-ray mirrors fabricated by electron beam, DC-, RF-magnetron sputtering and ion beam sputtering deposition," in X-Ray Multilayers for Diffractometers, Monochromators, and Spectrometers, F. E. Christensen, ed., Proc. SPIE 984, 140-148 (1988).

13. T. W. Barbee, J. C. Rife, W. R. Hunter, M. P. Kowalski, R. G. Cruddace, and J. F. Seely, "Long-term stability of a Mo/Si multilayer structure,” Appl. Opt. 32, 4852-4854 (1993).

14. K. Holloway, K. Ba Do, and R. Sinclair, "Interfacial reactions on annealing molybdenum-silicon multilayers," J. Appl. Phys. 65, 474-80 (1989).

15. D. G. Steams, R. S. Rosen, and S. P. Vernon, "Fabrication of high-reflectance Mo-Si multilayer mirrors by planarmagnetron sputtering," J. Vac. Sci. Technol. A 9, 2662-2669 (1991).

16. C. Khan-Malek, J. Susini, A. Madouri, M. Ouahabi, R. Rivoira, F. R. Ladan, Y. Lepetre, and R. Barchewitz, "Semitransparent soft X-ray multilayer mirrors,” Opt. Eng. 29, 597-602 (1990).

17. S. Hubert, Ph. Zeitoun, É. Béchir, D. Benredjem, F. Bridou, A. Calisti, F. Delmotte, M. Idir, G. de Lachèze-Murel, S. Le Pape, M. F. Ravet, D. Ros, L. Vanbostal, and S. Hubert, "Line shape measurement of a Ni-like silver X-ray laser by mean of soft X-ray Fourier-transform spectroscopy," submitted to Phys. Rev. A. 